\title{
USING THE INVERSE SOLVER “INVERSE” IN PAM-STAMP 2G TO ASSESS THE FORMABILITY OF A PART
}

\author{
A. A. Ershov ${ }^{1}$ and Yu. N. Loginov ${ }^{2}$ \\ This article discusses the possibility of using a finite-element-based inverse design method to evaluate a \\ technology for making parts by cold sheet stamping. It examines the process of reengineering in the software \\ suite PAM-STAMP. The results that are obtained provide information on the development of the product and \\ different pre-computed fields: stress-strain state, thickness reductions, defects, etc.
}

UDC 621.983:669.51-7

Keywords: PAM-STAMP, ESI-Group, development, finite-element method, inverse design.

When preparations are being made for manufacturing operations, having information on the required shape of the semifinished product, its stress-strain state, the reductions that are to be made in its thickness, and any surface defects that are present can greatly expedite the process of obtaining the finished product. Both the designer of the product and the production-process engineer can evaluate these parameters qualitatively immediately after the construction of a CAD model in order to validate the planning decisions that are made.

Developing the given part in PAM-INVERSE (a module of the program PAM-STAMP 2G, developed by the company ESI-Group ${ }^{\odot}$ ) makes it possible to obtain the shape and dimensions of the initial semifinished product before the stamping operation. In the course of solving the inverse problem, the user of the software also evaluates the stress-strain state of the metal and the reductions in its thickness. Having such information in turn makes it possible to obtain a preliminary estimate of the practicability of the shaping operation while the part is still in the design stage.

In this article, we take the results of calculations that are performed in PAM-STAMP 2G with the PAM-Inverse solver (and which produce a developed view of the part and provide a preliminary estimate of the theoretical fields) and we compare those results with the results obtained by using the main solver in PAM-Autostamp. The latter solver gives a more precise evaluation of all of the theoretical fields. The objective of our investigation is to explore the capabilities of the inverse solver and evaluate its use for analyzing the process of making complex products. As an example, we analyze results obtained in the stamping of two products used in the automobile industry (Fig. 1).

The actual process of producing each of these parts includes the operations of layout, clamping, and stamping. Individual data packets are created for each of these operations in preparation for calculations with the main solver of PAM-Autostamp. The packets include all of the input parameters of the production process that are needed to perform the calculations as accurately as possible for each stage of the process. Performing the calculations with the solver PAM-Inverse simplifies the formulation of the problem: the most important factors are modeling speed and ensuring a rapid transition from the form of the finished product to the form of the semifinished product. However, even though the formulation of the problem has been simplified, it still accounts for such parameters as the properties of the material, the anisotropy of its properties, the initial thickness of the semifinished product, the direction of motion of the tool, and cutting operations that might

\footnotetext{
$\overline{1}$ PLM Ural - Delkam Ural Company Group, Ekaterinburg, Russia; e-mail: eaa@ delcam-ural.ru.

${ }^{2}$ Yeltsin Ural Federal University (UrFU), Ekaterinburg, Russia.
}

Translated from Metallurg, No. 5, pp. 24-28, May, 2013. Original article submitted May 6, 2013. 

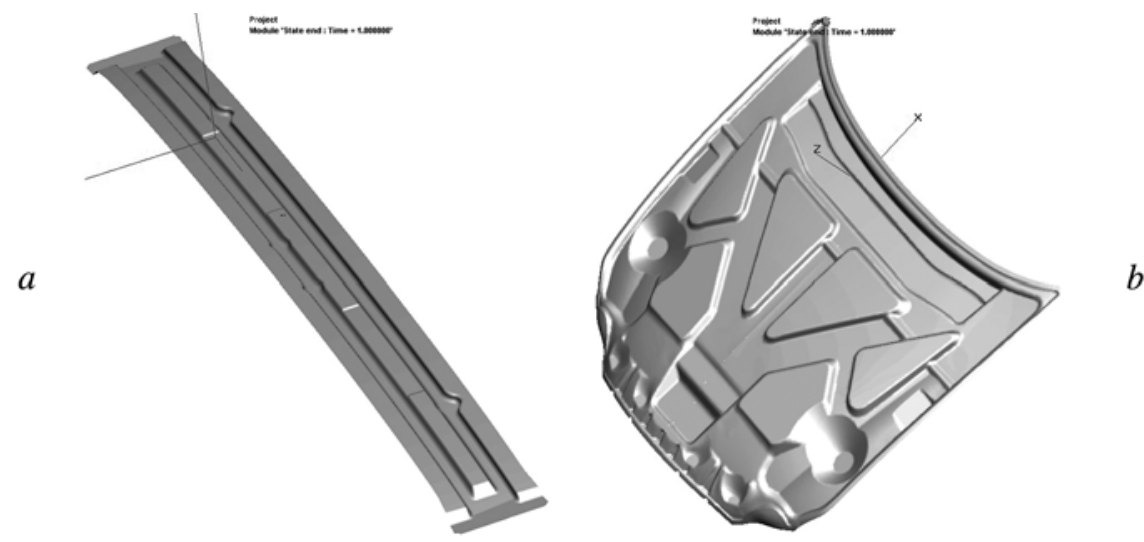

Fig. 1. Automotive parts being analyzed: $a$ ) element of a radiator side column; $b$ ) inside part of a hood.
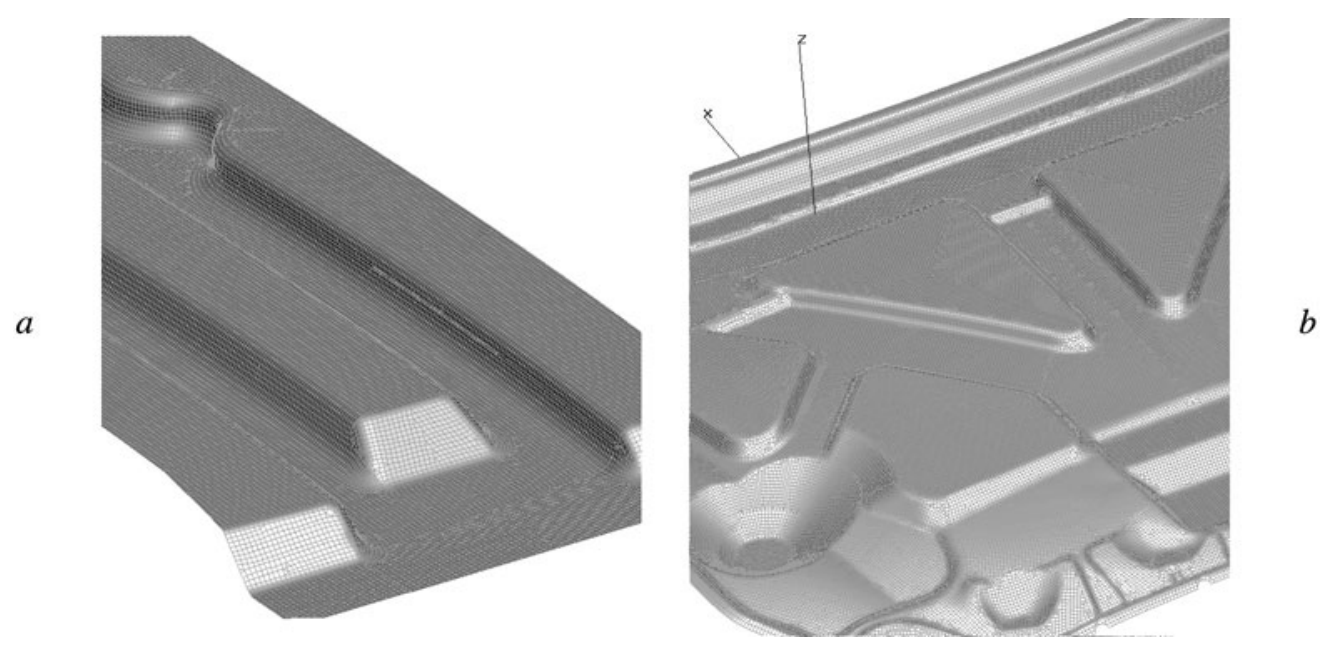

Fig. 2. Resulting FE-grids of the parts imported into the module that performs inverse design calculations: a) element of radiator side column; $b$ ) inside part of hood.

be performed in the actual production process (it is necessary to plot curves showing the paths along which the product could be cut).

Either the CAD-geometry of the product or its completed finite-element (FE) grid is imported at the beginning of preparations for performing the inverse calculations. Figure 2 shows imported FE-grids of the products being analyzed here.

The following initial parameters of the semifinished product were chosen for each part in formulating the problem: the initial thickness of the semifinished product for making the element of the radiator side column was $0.90 \mathrm{~mm}$; the material of the parts was steel $08 \mathrm{kp}$; the thickness of the hood was $0.65 \mathrm{~mm}$; the material of this part was aluminum.

The problem is formulated by means of a ready-to-use macro developed for the PAM-Inverse solver. Figure 3 shows the macro's parameters that are assigned to design a hood part and the properties tree that is ultimately obtained. The parameters for modeling the fabrication of the radiator-side-column element are similarly assigned.

The parameters of the solver are adjusted after the problem has been formulated. The PAM-Inverse solver makes it possible to use an SMP (shared memory process) - parallel computing - and thus ensure that the problem is solved at a high speed. The modeling operation takes several minutes, the exact time depending on the ultimate size of the grid elements and the complexity of the part. 

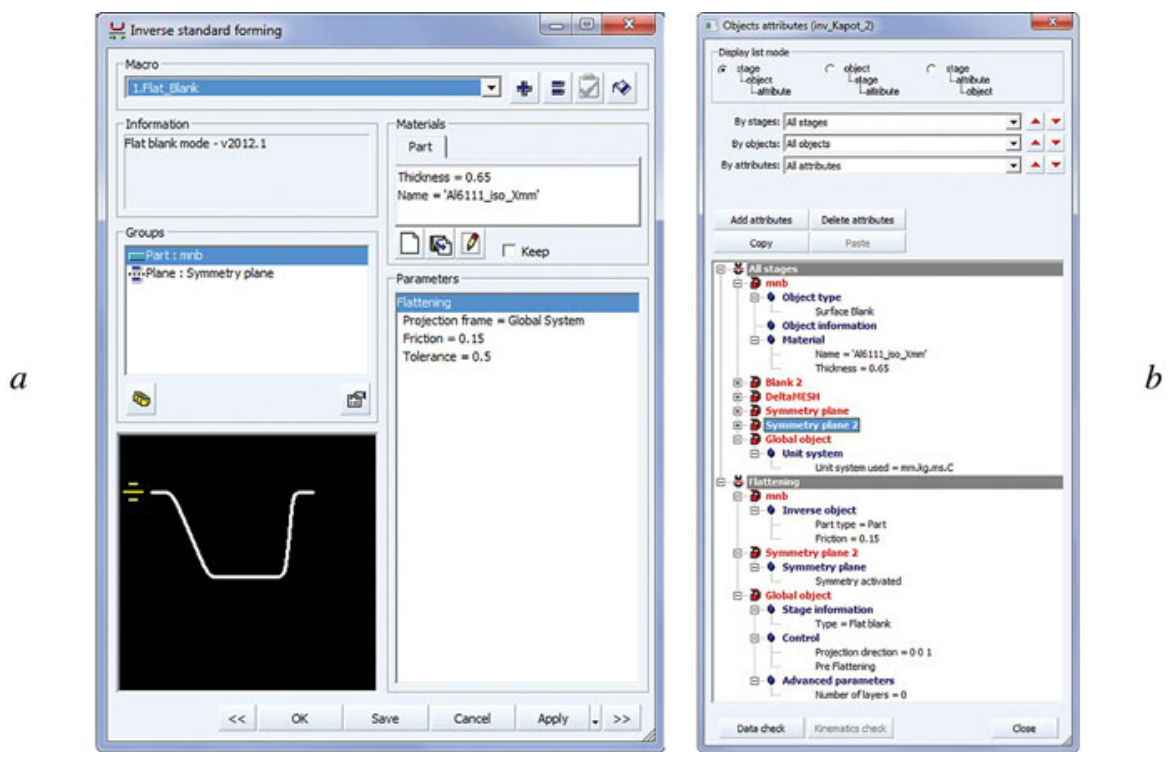

Fig. 3. Parameters of the macro used for calculation of the inverse problem for a hood part (a) and the design properties tree $(b)$.

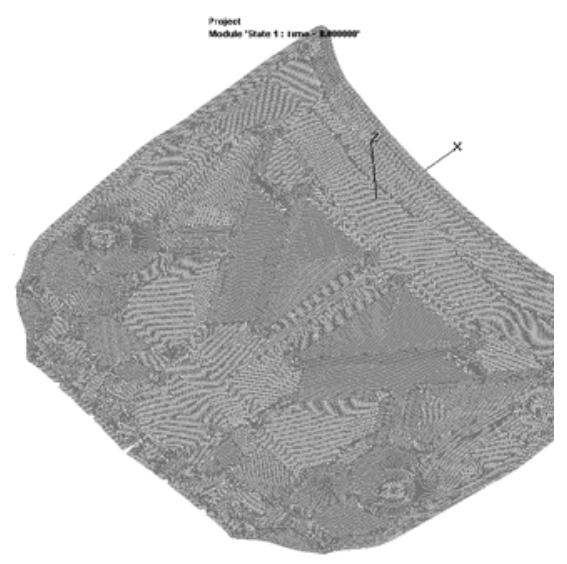

Fig. 4. Developed view of the hood interior obtained after calculations.

Data for performing calculations with the use of the main solver of PAM-Autostamp were obtained earlier, and the results of those calculations were compared to the actual part (the formulation of the problem in this case is not described in detail here).

After performing the modeling with the use of the inverse solver, we analyzed the results and compared them to the other results. Figure 4 shows the developed view of the internal portion of the hood part, which can be used to obtain the ideal (without additional machining) part in accordance with the calculations.

Figure 5 shows the distribution obtained for the thickness reductions inside the hood when the calculations are performed with the PAM-Inverse solver (Fig. 5a) and the PAM-Autostamp solver (Fig. 5b). The thickness reductions range up to $20 \%$ (PAM-Inverse) and 25\% (PAM-Autostamp), the difference between the values obtained in these two cases being $20 \%$. The character of the distributions is largely the same in each case, even though higher values are obtained with the use of the main solver.

Fig. 6, I shows the cumulative strain in the hood when calculations are performed with the two solvers. The maximum value is 0.26 when the inverse solver is used and 0.30 when the main solver is used. Thus, the difference is $13 \%$. The local 

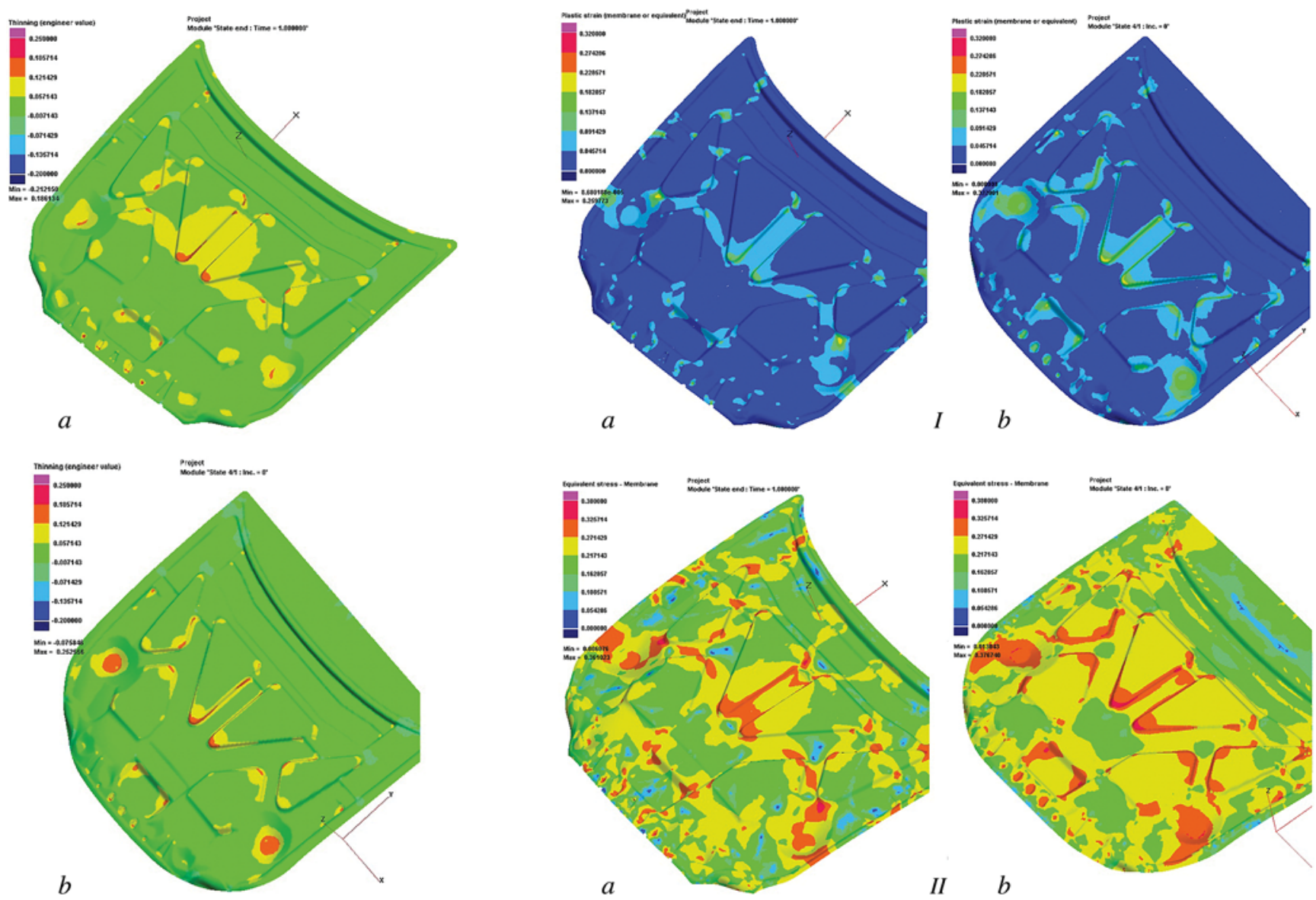

Fig. 5. Distribution of the reductions in the thickness of the inside part of the hood when the calculations are performed using the PAMInverse solver $(a)$ and the PAM-Autostamp solver $(b)$.
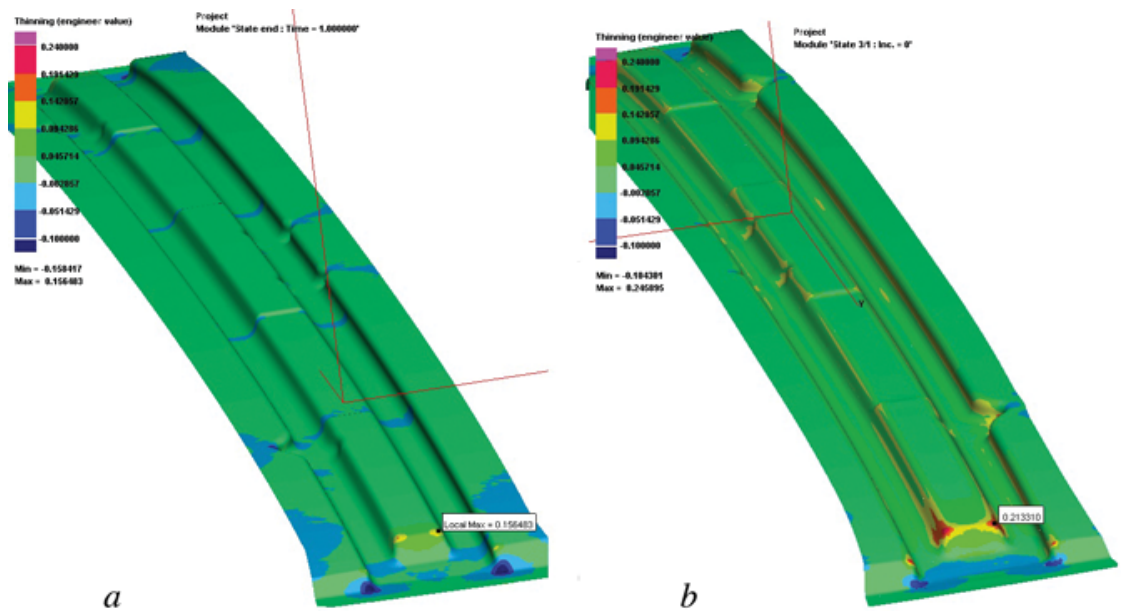

Fig. 7. Distribution of the thickness reductions in the radiator-side-column element when calculations are performed with the PAM-Inverse solver $(a)$ and the PAM-Autostamp solver $(b)$. 

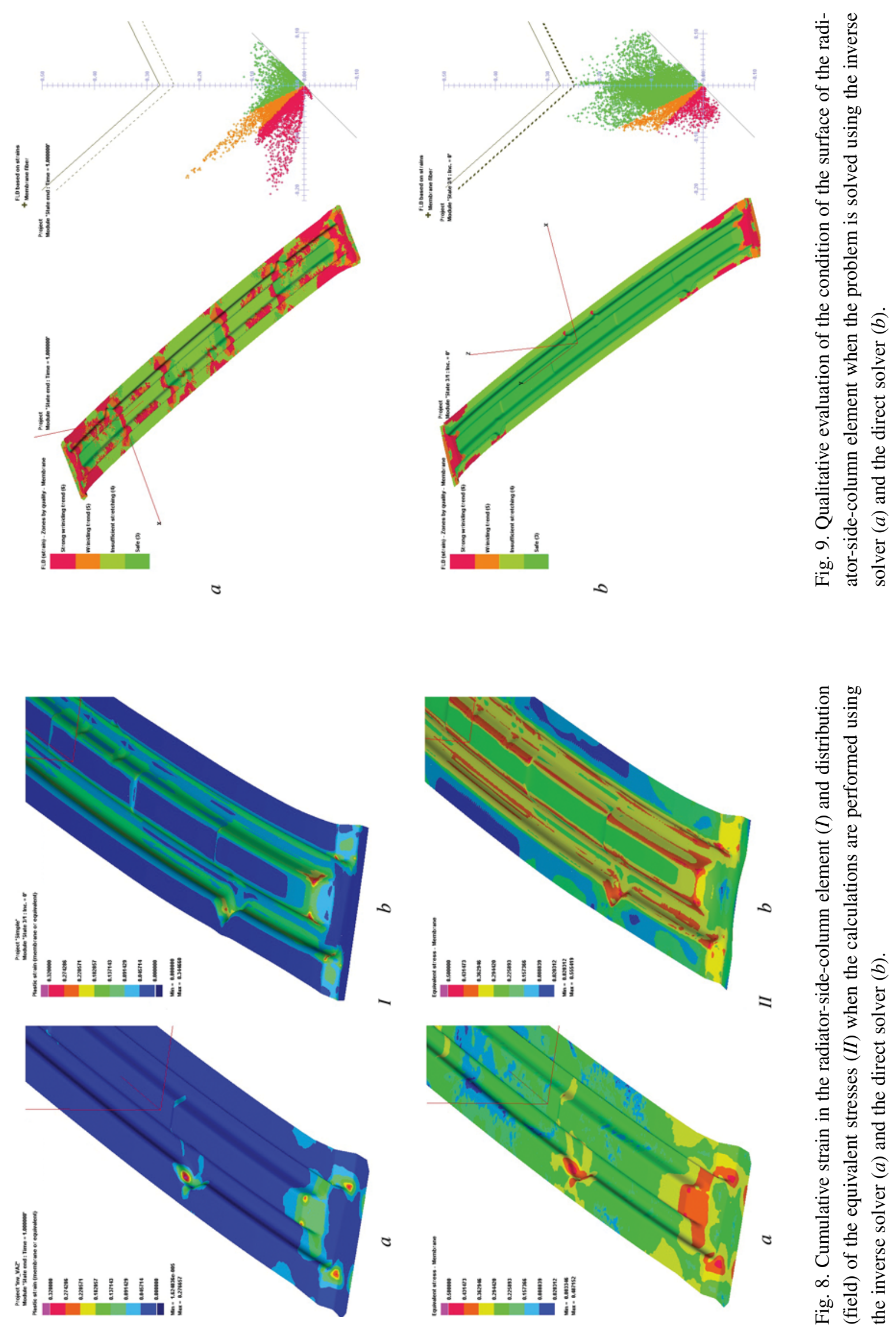
regions where the strains are greatest agree with a high degree of accuracy. The values calculated in the inverse solver are again lower that the values obtained in the main solver.

The strain can also be used to evaluate the stress level in the part. The stress distribution in the part (the field of equivalent stresses) was similar in each case (Fig. 6, II), although it is apparent that the stress level determined with the inverse solver is somewhat lower than that found with the main solver and that the values which were obtained provide only a qualitative estimate. The difference between the maximum values is $4 \%$ (approximately 361 and $376 \mathrm{MPa}$, respectively).

The following results were obtained for the radiator-side-column element by comparing the thickness reductions obtained using the PAM-Inverse solver (Fig. 7a) and the PAM-Autostamp solver (Fig. 7b). The reductions reach 16\% (PAMInverse) and 24\% (PAM-Autostamp). The largest reductions are local in character and are determined accurately with the inverse solver. However, the large difference between the maximum thickness reductions (at the given location) - which is on the order of $33 \%$ - requires more detailed study during the subsequent design of the part.

Figure $8, I$ shows the cumulative strain in the product when the two solvers are used to perform the calculations. The maximum value is 0.28 when the inverse solver is used and 0.34 when the main solver is used. Thus, the difference is $20 \%$. It is apparent that the inverse solver is less effective in determining the strain along the edges, where the elongation is greatest - there is no information on the bending of the material in these areas of the part. The largest stress concentrators were shown to be in the corner regions and the locations where the part undergoes reverse bending.

Figure 8,II shows the level of the equivalent stresses that corresponds to the cumulative strain in the part. The stress distribution obtained in the part with each solver differs appreciably for the same given scale: the maximum value obtained is $490 \mathrm{MPa}$ with the inverse solver and $560 \mathrm{MPa}$ with the main solver (difference of 12.5\%). It can be seen that there are no local concentrators at the sites where the part undergoes reverse bending - along the part's edges.

Evaluation of the quality of the surface by means of the diagram which describes the limiting formability of the given part shows that the sites where fold-type defects are more likely to be formed are much more widespread according to the inverse solver than according to the main solver and results obtained from a subsequent experiment (Fig. 9). The calculated results are most accurate in the vicinity of the corrugations located at the edges of the product. No folds were formed on the surface of the product in its central part; thus, the inverse solver gave a somewhat exaggerated picture of the defects actually formed on the surface.

Comparison of the results calculated with the two solvers leads to the following conclusions.

The above data calculated for the main theoretical fields with the use of an inverse solver show that although the results on the locations of the stress concentrators, the largest thickness reductions, and possible surface defects differ somewhat from the results obtained with a direct solver, these differences are probably connected with the lack of precise data on the process. The average difference between the results obtained with the PAM-Autostamp and PAM-Inverse solvers is $26 \%$ for the thickness reductions, $16 \%$ for the strains, and $7 \%$ for the equivalent stresses. Results calculated with the inverse solver make it possible to qualitatively evaluate the likely locations of defects, stress concentrators, etc., although the values found for most of the indices are underestimates. This should be taken into consideration and an approximate percentage error should be assigned to them. In addition, the PAM-inverse solver does not provide probabilistic data on the springback of the given part. Thus, the tool that is used to shape it may require some modification.

On the whole, the PAM-Inverse solver makes it possible to save time and money in preparing a part for production and facilitates the dialogue between the designer and the process engineer. 